\title{
A Review on the Development of Embodied Presentation Agents and their Application Fields
}

\author{
Thomas Rist ${ }^{1}$, Elisabeth André ${ }^{2}$, Stephan Baldes ${ }^{1}$, Patrick Gebhard ${ }^{1}$, \\ Martin Klesen ${ }^{1}$, Michael Kipp ${ }^{1}$, Peter Rist ${ }^{1}$, and Markus Schmitt ${ }^{1}$ \\ 1 DFKI GmbH, Stuhlsatzenhausweg 3, D-66123 Saarbrücken, Germany. \\ \{rist, baldes, patrick.gebhard,klesen, kipp,mschmitt\}@dfki.de, \\ pit@blinx.de \\ 2 Augsburg University, Eichleitnerstr. 30, D-86135 Augsburg, Germany \\ andre@informatik.uni-augsburg.de
}

Summary. Embodied conversational agents provide a promising option for presenting information to users. This contribution revisits a number of past and ongoing systems with animated characters that have been developed at DFKI. While in all systems the purpose of using characters is to convey information to the user, there are significant variations in the style of presentation and the assumed conversational setting. The spectrum of systems include systems that feature a single, TV-style presentation agent, dialogue systems, as well as systems that deploy multiple interactive characters. We also provide a technical view on these systems and sketch the underlying system architectures of each sample system.

\section{Introduction}

The last decade has seen a general trend in HCI to make human-computer dialogue more like human-human dialogue. Computers are ever less viewed as tools and ever more as partners or assistants to whom tasks may be delegated. Trying to imitate the skills of human presenters, some R\&D projects have begun to deploy animated agents (or characters) in wide range of different application areas including e-Commerce, entertainment, personal assistants, electronic learning and training environments. Based either on cartoon drawings, recorded video images of persons, or 3D body models, such agents provide a promising option for interface development as they draw on communication and interaction styles humans are well familiar with.

Starting in the mid 1990s with the development of the presentation agent "PPP Persona", DFKI has contributed to this area of research by introducing a plan-based approach to automate the process of writing scripts that control and constrain the behavior of presentation agents. Since then this approach 
has been successfully applied to build a number of applications in which information is conveyed either by a single presenter or by a team of presentation agents. Looking at past and current projects conducted at DFKI we observe an ongoing and manifold evolution of character-based presentation systems.

First of all, there is an obvious maturation of a character's visual and audible appearance. This maturation has become possible mainly due to more powerful but nevertheless affordable graphics hardware as well as due to advances in animation and speech synthesis technology. While many early interface agents were animated on the basis of a relatively small number of handdrawn 2D cartoons, rich 3D-body models can now be animated in real-time, and improved speech synthesis enables voice qualities that sound less robotic. Improved audio-visual attractiveness alone, however, is only one ingredient for making better virtual characters. In addition, the success of characters in terms of user appreciation and added value in information mediation tasks very much depends on other factors too, including a character's role, competence, and communicative skills relative to an application, and its ability to present itself as believable virtual personality. With a focus on structure and complexity of the conversational setting that a character faces, this contribution revisits a number of past and ongoing systems developed at DFKI, discusses impacts on the architectural design, and provides references to related systems.

\section{From TV-style Presenters to Interactive Performances}

The choice of domain, tasks, and conversational setting imposes constraints on any prototype development. For instance, in the area of intelligent information presentation with animated characters we observe an ongoing evolution of systems as illustrated in Fig. 1. The first setting refers to applications in which a single character is deployed to present information. From the point of view of the user a generated presentation appears quite similar to watching a TVnews speaker or to the display of a video clip because no interaction is foreseen at display time. In contrast, the second setting is typical for applications with characters that are able to converse with a user in some sort of a dialogue (e.g. via spoken or typed natural language, or based on dynamically configured menus). Moving on to the third setting actually means a shift from a faceto-face character-user setting to a user-as-observer setting. That is, two or more characters talk to each other on the screen to convey information to the observing audience. However, no user intervention is foreseen during a performance. This is in contrast to the fourth scenario where we have an open multi-party dialogue setting which allows for both reactive and proactive user participation.

Technically speaking the fourth scenario is quite challenging since one has to resolve on an operational level the conflict between predestination and freedom of interaction. To complicate things even further, one can think of 
multi-party settings with multiple characters and multiple users. However, up to now such settings remain a big challenge since in this case the characters must also be able to overhear and understand conversations among the human partners.

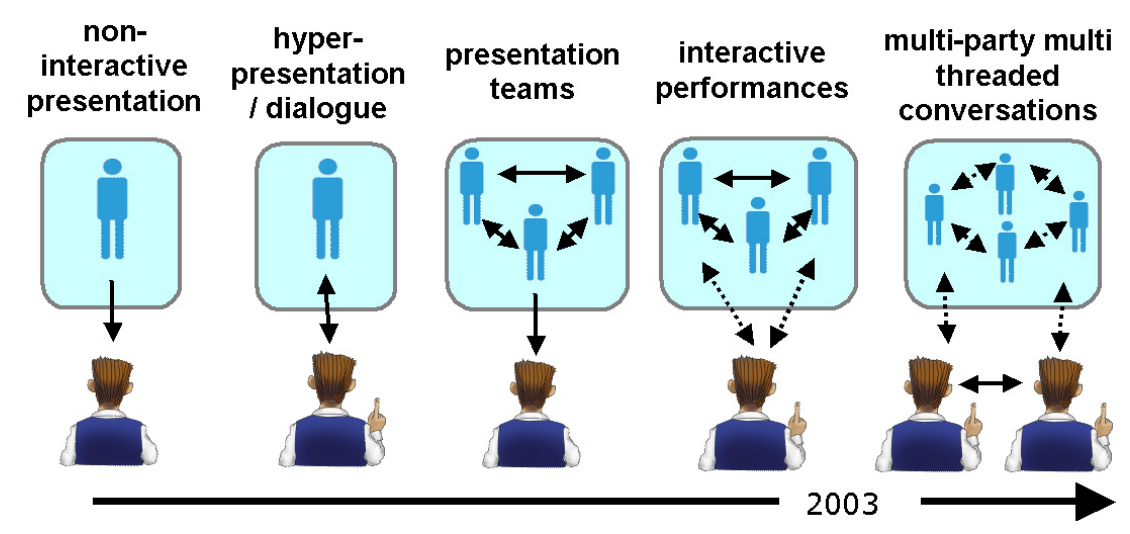

Fig. 1. Character applications with different conversational settings

\section{Non-interactive Presenters}

The Internet boost in the mid 1990s has inspired researchers as well as companies to develop applications in which a virtual character takes on the role of a kind of personalized TV-presenter who informs and entertains the user. A popular example is the virtual news reader Ananova (www.ananova.com) that reads news scripts live from ITN, a British broadcaster producing news.

Concerning the control of such virtual presenters one can distinguish between manually scripted characters and systems that work out presentation scripts automatically. To support manual scripting, a number of dedicated markup languages have been proposed, some of which are presented in detail in Part 1 of the current volume. Since manual scripting can be tedious and error-prone, several attempts have been made to automate the character scripting process either partially or completely. Examples include Noma's and Badler's virtual weather reporter (Noma and Badler [33]), Thalmann's and Kalra's [46] TV presenter and the systems Byrne (Binsted and Luke [7]) and Mike (Matsubara et al. [31]) that deploy a talking head to comment on matches of the RoboCup simulation league (Kitano et al. [21]). Further examples of related systems can be found in other chapters of the current book. A DFKI system that falls into this category was developed in the PPP (Personalized Plan-based Presenter) project (André and Rist [1], Rist et al. [39]). 


\subsection{PPP Persona}

PPP-Persona was designed as a personalized presentation agent that provides multimodal instructions for the operation of technical devices. For instance, to explain how to switch on a technical device, PPP-Persona may show the user a picture of the device, point to the on-off switch while giving verbal instructions on how to manipulate the switch (cf. left-hand side of Fig. 2).

From a functional perspective, PPP Persona receives as input presentation goals (PG) and generation parameters (GP), such as the user's level of expertise and time constraints for the duration of the presentation to be generated. As output, the system delivers a specification of a multimedia presentation, called presentation script. Such scripts are forwarded to a dedicated player engine responsible for the synchronized display of all involved media objects including possibly animated illustrations of domain objects, text elements, as well as character animations and verbal speech output.
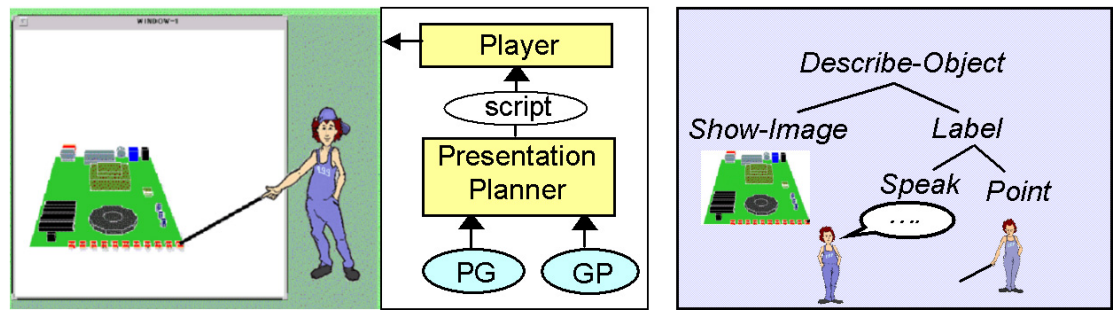

Fig. 2. PPP Persona: Screenshot and architecture (left). Excerpt of a presentation plan (right)

In PPP, we formalized action sequences for composing multimedia material and designing scripts for presenting this material to the user as operators of a planning system. Starting from a complex presentation goal, (e.g. to describe a technical device) the planner recursively performs a hierarchical goal decomposition until all subgoals have been expanded to elementary production, retrieval or presentation tasks. A portion of a hierarchical presentation plan is shown in the right-hand part of Fig. 2. The operators of the PPP system represent tactical knowledge about how to achieve presentation goals by means of a multimedia presentation including a character as an additional presentation device. Therefore, the operators are formulated from the point of view of a director who orchestrates the interplay of the character with the display of all other media objects. As to modularization of the planning mechanism, PPP uses just one planner for script generation. Details on the planning approach can be found in André et al. [3]. 


\section{Conversational Characters}

A great deal of contemporary systems aims at emulating aspects of a face-toface interaction in settings where a user faces a virtual conversation partner. Differences among systems concern both available input modalities as well as output modalities.

\subsection{Characters with Restricted Input Understanding Capabilities}

In the simplest case, user input is handled in a rather restricted and controlled fashion. An example of such a system was developed in the AiA (Adaptive Communication Assistant for Effective Infobahn Access) project (André et al. [3]). The corresponding AiA system features a personal embodied travel agent whose task is to collect, structure, and present information about a travel destination while taking into account the individual user profile. A screenshot of the system is shown in the left-hand part of Fig. 3. During the presentation phase the AiA agent solicited input from the user only at some distinct stages mainly to let the user choose between several options about what to present next or on what to elaborate in more detail. Thus, presentations generated by the AiA systems can be regarded as a special type of hypermedia presentations.

In contrast, the stock agent "Rudi" (see right-hand part of Fig. 3) engages with the user in a natural language conversation. Being connected to several online stock servers, a user can chat with Rudi about the latest developments of shares. The user "talks" to Rudi by typing natural language expressions into a text input widget while Rudi will talk to the user either by voice output or likewise through speech bubbles. Also, Rudi makes use of gestures and facial displays that accompany his verbal utterances or are even used stand-alone to convey a communicative goal non-verbally.

As shown in the sketched architectures, AiA's as well as Rudi's internal machinery is quite similar to that of the PPP Persona. However, both systems also comprise a component to process user input. In case of the AiA system, selected menu options correspond directly to presentation goals or new settings of presentation parameters. In the case of the stock agent Rudi a shallow natural language analysis is performed. Deploying the ALICE framework for programming chat robots (www.alicebot.org/), pattern matching rules are applied to derive requests for new presentation goals and new settings of presentation parameters from a user's input message.

\subsection{Characters that Engage in Multimodal Conversations}

To emulate more closely face-to-face dialogue settings among humans it is desirable to avoid asymmetries in communication channels that are available to a human and a character. 

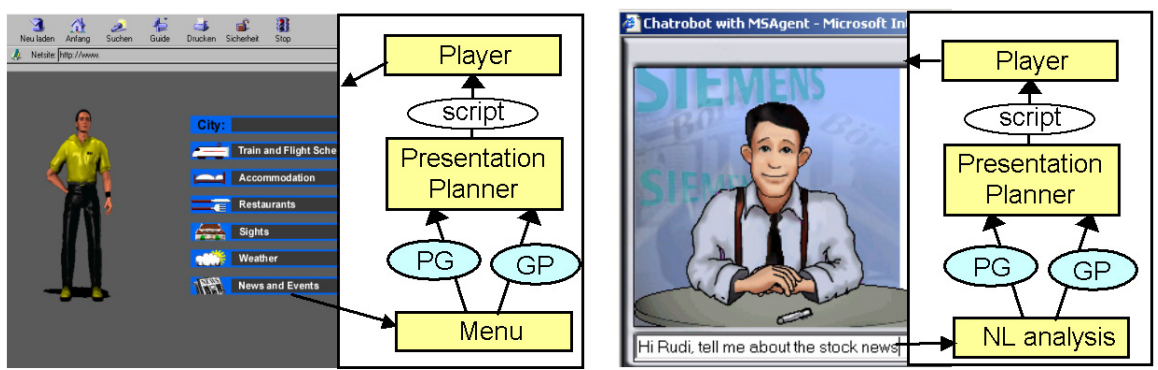

Fig. 3. Screenshot and architecture of the AiA travel agent (left) and stock agent Rudi (right). In contrast to the PPP architecture (cf. Fig. 2), the AiA and Rudi both comprise components for analyzing user input, such as menu-based follow-up questions and typed natural language queries

The ability of a character to engage with a human in an unconstrained spoken natural language conversation is most desirable but also very difficult and therefore will remain a great challenge for years even though considerable progress has been made in the last decade in speech recognition, synthesis and spoken dialogue systems. On the other hand, restricted spoken-dialogue agents are technically feasible and may suffice their purposes in some domains. For instance, the virtual receptionist Cyberella (Gebhard [13]) understands a limited number of questions concerning typical information requests of visitors coming to DFKI. For instance, a visitor may ask her for directions to get to a certain office. From an architectural point of view, Cyberella shares similarities with the Rudi stock agent except that this time aural user input is accepted and processed by a speech recognizer to obtain a text string which is then processed further by means of a pattern-based natural language interpreter.

More ambitious attempts to overcome asymmetries in available communication channels have been made in the SmartKom project (Wahlster et al. [52]). Firstly, the interface character Smartakus comprises a more sophisticated spoken dialogue subsystem. Secondly, Smartakus has also a "visual sense" that enables it to recognize and understand pointing gestures of the user. Thirdly, its visual sense allows the character to read a limited number of emotional expressions from the user's face. Recognized user emotions can be used to disambiguate speech input and to generate affective responses. Figure 4 shows from left to right the agent Smartakus acting as a TV-program advisor, and two shots of the SmartKom installation for public spaces. As shown by the close-up, Smartakus interprets multimodal user input, such as verbal utterances that are accompanied by pointing gestures.

Even though projects like SmartKom break new ground in the area of multimodal human-character interaction, the emulation of multimodal humanhuman face-to-face communication remains a long-term challenge. 


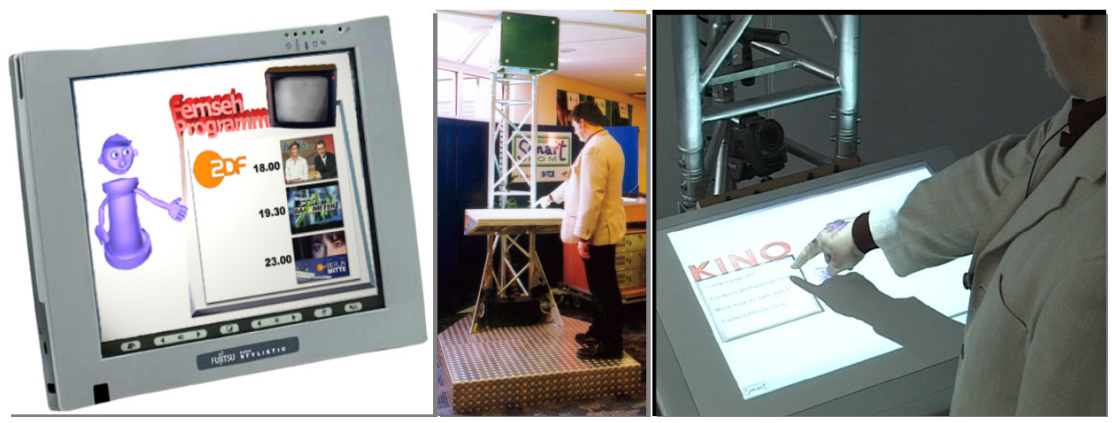

Fig. 4. Shots from the SmartKom system. In SmartKom the user engages in a multimodal conversation with the agent Smartakus.

\subsection{Other Conversational Agents}

Quite a number of commercial sites try to boost their web presence by adding an embodied conversational character. Similar to the stock agent Rudi, the user can "talk" to the character by typing NL expressions into a text-input widget. In most cases, however, the conversational skills of these characters are limited to that of a pattern-based chat robot. In the best case, such systems manage to map user input to related contents of the web site. The virtual chat agent Cybelle (www.agentland.com/) and the agent Aisa (www.smart.com) are examples of this kind. In the worst case, a conversation with such a character is neither informative nor entertaining. Needless to say that such characters are likely to be perceived by a user as useless if not annoying.

In contrast, most research prototypes of embodied conversational characters are instances of complex multimodal dialogue systems, though the focus is usually on the generation of sophisticated multimodal expressions by which a character should respond to user input. Prominent examples include the Internet Advisor Cosmo (Lester et al. [25]), the REA real estate agent (Cassell et al. [10]), and the GRETA medical advisor (Pelachaud et al. [34]) whereby the last two systems rely on sophisticated models of multimodal communication.

Besides building concrete agents, researchers focus on the development of application-independent tools for the implementation of virtual dialog partners. Well-known examples of this type include the CSLI toolkit (Cohen et al. [12]) and the Collagen system (Rich et al. [37]) which is based on a model of collaboration between a human and a computer agent.

\section{Presentation Teams}

There are situations in which direct agent-user communication is not necessarily the most effective and most convenient way to present information. Inspired by the evolution of TV commercials over the past 40 years, our group 
has discovered role-plays with synthetic characters as a promising format for presenting information. A typical TV commercial of the early days featured a sales person who presented a product by enumerating its positive features quite similar to what synthetic characters do on web pages today. On TV, however, this format has been almost completely replaced by formats that draw on the concept of short, entertaining scenes. Typically, such performances embed product information into a narrative context that involves two or more human actors. Episodic formats offer a much richer basis compared to the plain enumeration of product features, and thus meet the commercial industry's high demand for originality.

A shift from settings with single presentation agents towards the use of presentation teams bears a number of advantages: First of all, they enrich the repertoire of modalities to convey information. For example, they allow a system to convey certain rhetorical relationships, such as pros and cons, in a more canonical manner. Furthermore, they can serve as a rhetorical device to reinforce beliefs. For instance, the same piece of information can be repeated in a less monotonous and perhaps more convincing manner simply by employing different agents to convey it. Furthermore, the single members of a presentation team can serve as indices, which help the user to organize the conveyed information. For instance, characters can convey meta-information, such as the origin of information, or they can present information from different points of view, e.g. from the point of view of a businessman or the point of view of a traveller. Last but not least, multiple characters allow us to convey social aspects, such as interpersonal relationships between emotional characters (see Prendinger and Ishizuka [36], Rist and Schmitt [41]).

\subsection{Simulated Car-sales Dialogs}

The eShowroom (also called "Inhabited Market Place") is an example of a system that employs presentation teams to convey information about products, such as cars, by performing role plays (André et al. [4]). The left-hand part of Fig. 5 shows the characters Tina and Ritchie engaging in a simulated car-sales dialogue.

The overall system's presentation goal is to provide the user with facts about a certain car. However, the presentation is neither just a mere enumeration of facts about the car, nor does it have a fixed course of dialogue between the agents. Rather, the eShowroom allows the user to specify prior to a presentation (a) the agents' roles, (b) their attitude towards the product, (c) some personality traits (extravert vs. introvert, agreeable vs. not agreeable), and (d) their interests about certain aspects relevant for cars (e.g. the car's relation to prestige, comfort, sportiness, friendliness to the environment, costs etc.). Based on these settings, a variety of different sales dialogues can be generated for the same product.

Similar to the PPP system a user specifies both a presentation goal and generation parameters prior to the presentation generation process. Since this 
time the behaviors of several characters have to be determined, a designer of such a system has the choice between taking a director's point of view or to adopt the self-scripting paradigm. In the first case, the task of the presentation planner is to work out one single script for the role play as a whole. Typically, such a script would include statements of the form: "character1 do $\mathrm{x}$; character2 do y". As shown in the depicted architecture of the eShowroom (left-hand part of Fig. 5), this system uses one centralized presentation planner to script the actions of the virtual seller and buyer agents in a car-sales dialogue.

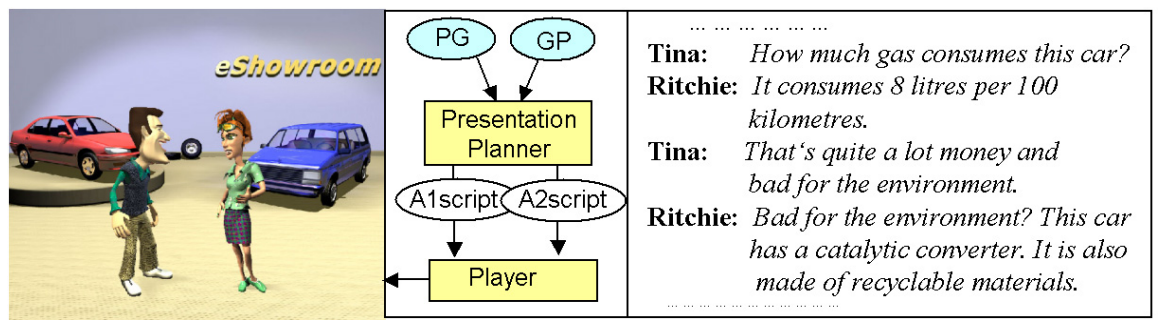

Fig. 5. Left: Screenshot and architecture of the eShowroom. Right: Excerpt of a "sales talk" between the buyer agent Tina and the seller agent Ritchie

\subsection{Reporter Teams}

On the other hand, there are a number of application fields for presentation agents where scripts for the agents cannot be worked out in advance since all or part of the information to be presented becomes available only at presentation runtime. Any kind of reportage or commentary of live data falls into this category. As an example, let us have a look at Rocco II (André et al. [5]), an automated live report system that generates commentaries for the simulator league of Robot World-Cup Soccer (Kitano et al. [21]). The right-hand part of Fig. 6 shows a screenshot of the system. In the upper window, two teams of software agents play a soccer game while being commented by Gerd and Matze, two virtual soccer fans sitting on a sofa and watching the game. As in the eShowroom system the user has the option of experimenting with different character profiles. For instance, the user may characterize Gerd and Matze by their personality and their sympathy for a certain team.

Unlike the agents in the car sales scenario, the RoboCup commentators have to comment on a rapidly changing environment. Since events on the soccer field evolve while time progresses, no global organization of the presentation is possible. Instead the commentators have to respond immediately to incoming data. Furthermore, they have to meet severe time constraints imposed by the flow of the game. In some cases, it might even be necessary for the commentators to interrupt themselves. For instance, if an important 


Gerd: $\begin{aligned} & \text { Wow, did you see this fantastic } \\ & \text { shot? }\end{aligned}$
Matze: Yeah but look where the ball
has gone.
Gerd: Oh no
Matze: The red team is going to make
$\begin{aligned} & \text { a counter attack. } \\ & \text { Matze: Come on, kick the ball }\end{aligned}$

Fig. 6. Left: Verbal comments by the soccer fans Gerd and Matze while watching a game. Right: Screenshot and architecture of the Gerd and Matze soccer commentary system

event (e.g. a goal kick) occurs, utterances are interrupted to communicate the new event as soon as possible. In such a situation, it is not possible to pre-script utterances. Instead scripting has to be done at runtime, e.g. either by a centralized script writing component or by the single agents themselves.

As shown by the architecture of the system Gerd \& Matze are realized as autonomous agents. That is, each agent has its own reactive planner and its own set of dialogue and commentator strategies. Dialogue contributions then result from autonomous characters trying to achieve their individual goals. The goal of the single commentators is to inform the viewer about ongoing events in the scene. In the current version of Rocco II, each commentator concentrates on the activities of a certain team. That is, there is an implicit agreement between the characters concerning the distribution of dialogue contributions. Responses to dialogue contributions of the other commentator are possible provided the speed of the game allows for it. Furthermore, the commentators may provide background information on the game and the involved teams. Concerning the commentary of the observed actions and events during the game, we assume that both commentators share all knowledge about the events on the soccer field which is provided by Rocco's incremental event recognition component (André et al. [2]). Assuming a discrete time model, at each increment of a time counter, the recognition component selects relevant events, formulates corresponding presentation tasks, and writes them into a buffer. In addition, the buffer contains presentation tasks that refer to the presentation of background information. If an event has been communicated or in case that the topicality of an event falls below a threshold, the corresponding presentation task is removed from the buffer again.

\subsection{Other Approaches with Multiple Characters}

Using multiple conversational characters that - rather than addressing the user directly - convey information to the user by talking loudly to each other is a concept that has been explored in a number of other contexts too. 
In some cases, these dialogues are manually scripted as in the Agneta \& Frida system (Höök et al. [15]) that incorporates narratives into a web environment by placing two characters on the user's desktop. These characters watch the user during the browsing process and make comments on the visited web pages. To facilitate the authoring of multimodal presentations with multiple characters, Ishizuka and colleagues [18] have developed the MPML scripting language. Details of MPML and a MPML authoring tool can be found in the current book.

Systems that aim at a simulation of conversations between humans usually automate at least parts of the generation process. Cassell and colleagues (Cassell et al. [9]) automatically create and animate dialogues between a bank teller and a bank employee with appropriate synchronized speech, intonation, facial expressions and hand gestures. Walker and colleagues (Walker et al. [53]) concentrate on the linguistic capabilities of computer characters (e.g. a waiter and a customer) and examine how social factors influence the semantic content, the syntactic form and the acoustic realization of conversations. The generation of their dialogues is essentially influenced by the power the listener has on the speaker and the social distance between them.

Mr. Bengo (Nitta et al. [32]) is a system for the resolution of disputes which employs three agents: a judge, a prosecutor and an attorney which is controlled by the user. The prosecutor and the attorney discuss the interpretation of legal rules. Finally, the judge decides on the winner.

Multiple characters have also been deployed in training applications. For instance, Rickel and Johnson [38] deploy characters in team training tasks where one character can represent an instructor while further characters may substitute missing team members.

\section{Presentation Teams that Represent Human Users}

In the eShowroom as well as in the Gerd \& Matze commentator system the members of the presentation team were virtual characters. In contrast, the members of presentation teams can also represent real humans. Examples of such applications are the systems Magic Monitor and Avatar Arena both developed at DFKI.

\subsection{Magic Monitor}

The Magic Monitor is a tool for illustrating message exchange in a multiuser conferencing system called Magic Lounge. Cartoon-style characters are used to represent different conversation partners (which may be humans or virtual conversational agents). The tool allows for the play-back of recorded message exchanges according to different structuring criteria, such as timelines or dialogue threads. The sequential nature of replaying recorder multiparty conversations is often somewhat long winding. However, the format can be useful for illustrating some crucial exchanges during a conversation. 
In the Magic Monitor the system plays a two-fold role. Firstly, it acts as a screen writer that determines how a conversation within the Magic Lounge should be played back by the single characters. Secondly, it is actively involved in the presentation through a facilitator agent that provides some meta information about recorded conversations. The screenshot in the middle of Fig. 7 shows a facilitator agent located in the center, while the other characters represent different users. As shown by the architecture of the Magic Monitor (left-hand side of Fig. 6), a centralized presentation planner determines the dialog script taking into account (a) the repository of recorded message exchanges, (b) a presentation goal that constrains the selection of message exchanges to be replayed, and (c) additional generation parameters, such as the assignment of characters to represent the communication partners.

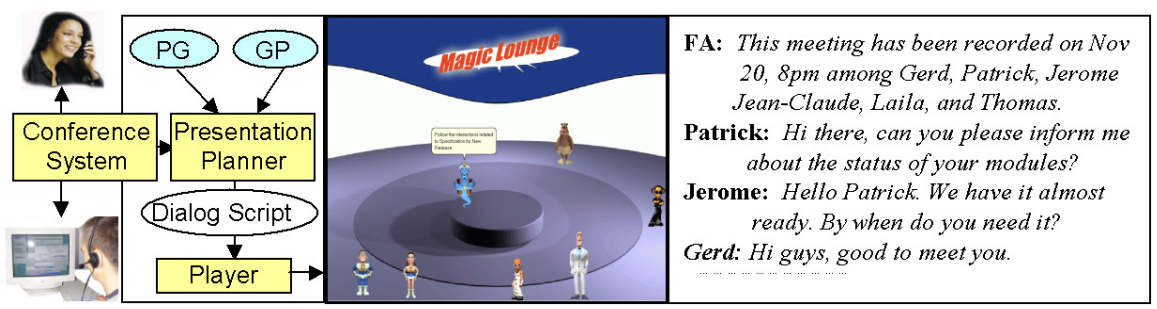

Fig. 7. Left: Architecture and screenshot of the Magic Monitor. Right: Excerpt of a replayed chat conversation. As an introduction the facilitator agent (FA) informs about time and participants of the conversation

The presentation planner's basic approach for message playback according to the time-line of occurrence comprises the following strategies:

1. have the facilitator announce the next message by referring to aspects, such as sender, date, time, topic and speech act;

2. have each character representing the sender of a message move to the center podium;

3. if a message is directed to a specific recipient, have that recipient move closer to the podium and play a "listen" animation;

4. have the message sender read the message while playing an animation that enforces the speech act associated with the message;

5. finally, have all characters move back to their assigned home positions.

Variations of the basic scheme have been defined to cope with messages, such as login, logout and registration, and to take into account various aspects, such as consecutive messages from the same user or the need for a more compact presentation. 


\subsection{Avatar Arena}

While the Magic Monitor replays recorded message exchanges of human communication partners, Avatar Arena simulates negotiation dialogs with affective, embodied conversational characters that are embedded in a social context. Somewhat similar to an arena, users send their delegates (avatars) to a virtual space where the avatars negotiate on behalf of their owners. Both result and process of a negotiation can be displayed to the users in form of a simulation using embodied conversational characters. Since the outcome of a delegated negotiation process can be unexpected, the user may wish to learn about how a certain negotiation result came about. In human-human negotiations this is of particular interest in cases where the result of a negotiation can not be explained on the basis of a solely rational argumentation but only if the social context and the personalities of the negotiating parties need to be considered as well.

Technically speaking, Avatar Arena can be conceived as a distributed n:1 client server architecture. While the server component provides the arena where the negotiation takes place, a client component allows a user to configure and instruct her/his avatar, and also to observe the negotiation process carried out on the server. To this end, the client receives a generated script of the overall negotiation dialogue for display (see right-hand part of Fig. 8).

Arena avatars negotiate on meeting appointments on behalf of human users. However, we have picked this domain just for the purpose of illustration and do not attempt to improve existing appointment scheduling tools. Rather, our research focus is on the simulation of the dynamics of social relationships among affective characters during negotiation dialogues. Avatar Arena serves as a test-bed to investigate and evaluate mind models of varying "cognitive complexity" for virtual characters that engage in negotiation dialogues. Our working hypothesis is that an increase of believability in the observable interactions among the characters will indeed require some higher extent of cognitive modeling. Our approach for assessing believability of negotiation dialogues is to show human observers several negotiation dialogues with virtual characters that differ in the number of psychological factors taken into account in a character's mind model. To this end Avatar Arena can simulate negotiation dialogues among avatars that vary in their modeled cognitive complexity.

In the simplest case, Arena avatars have only some domain knowledge about appointment dates and rudimentary conversational skills that enable them to propose meeting dates, and to accept or reject proposals based on their personal calendar entries. Since characters of this type can only talk about availability or not-availability concerning proposals for meeting dates, the generated negotiation dialogues have a strong flavor of being nothing more than a mere trace log-file as known from multi-agent expert systems.

In a second step we consider characters that have attitudes concerning already scheduled meeting dates, and new dates to be negotiated. That is, we 


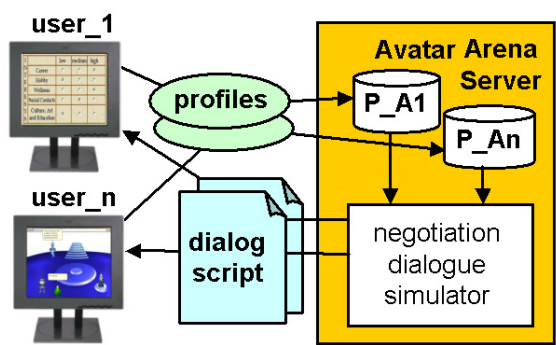

A1: We need to arrange a working meeting.

A1: I'm available the day after tomorrow.

A2: I'm available then.

A3: I'm not available then. I have a meeting with my family the day after tomorrow.

A2: Can't you postpone this? Our meeting is perhaps somewhat more important.

A3: My family is more important than work. A1: I 'm quite pleased to hear that from you.

Fig. 8. Left: Architecture of Avatar Arena. Right: Excerpt of a meeting negotiation dialog between three avatars. Some of the utterances reflect that the avatars have attitudes towards activities associated with meeting dates and also attitudes towards other avatars

allow the users to assign importance values to appointments that the avatars should take into account in a negotiation. This extension to the avatar's mind model has an impact on the negotiation process and is also reflected by the resulting dialogs. Firstly, the attitudes can be considered to determine importance values or priorities for meeting dates under discussion. In turn, such a priority order provides a rational criterion for an avatar to determine its willingness to reschedule an already fixed appointment. That is, when a date for the new appointment is proposed, the avatar compares the importance value assigned to the new meeting activity with the importance value of the already scheduled activity. For instance, if an avatar believes in career and does not care much about social contacts, it may be willing to postpone a holiday trip with friends and attend a conference instead. Furthermore, attitudes towards meeting dates and the activities associated with these dates give the avatars a rational basis for making justifications in a negotiation dialog. For instance, an avatar can argue that it won't be willing to reschedule a medical treatment in favor of a working meeting because of its particular attitudes. In addition, the importance value assigned to an already scheduled meeting date can be used as a criterion for deciding whether to provide a justification at all. However, this version of Avatar Arena neglects the fact that in a group setting, the single participants have attitudes towards their negotiation partners too. Moreover, both attitudes towards subject matters, such as meeting activities, as well as attitudes towards dialogue partners are subject to change in a negotiation process.

This motivates a third version of Avatar Arena which enables a user to specify her/his attitudes towards other persons (which are assumed to be represented by their avatars in a negotiation process). In this version of the system the avatars consider: (i) their own attitudes towards meeting dates, (ii) attitudes towards other avatars, and (iii) believes about the other avatars' attitudes towards meeting dates. Again, this increase in the avatars' "cognitive complexity" leads to noticeable differences in the negotiation dialogues. In particular, this version of Avatar Arena emulates some aspects of group dynamics 
(in terms of changing attitudes) as known from negotiation dialogues among humans. For instance, the avatars are now able to make personal comments on justifications of other dialogue partners, and to reply to personal comments made by others. The underlying approach to model group dynamic aspects is inspired by socio-psychological theories of cognitive consistency, such as Congruity Theory by Osgood and Tannenbaum. Details of this approach can be found in Rist and Schmitt [41].

\subsection{Other Applications with Avatars}

To represent users through embodied characters in virtual space is an old idea for which the term "Avatar" has been coined. An obvious application area for avatars are multi-party gatherings and conversations that take place in virtual space. Graphical chat systems in which users have either 2D or 3D representations fall into this category. Sample systems are, for instance Comic Chat (Kurlander et al. [23]) and V-Chat (Smith et al. [43]) both developed at Microsoft Research. The avatars in these systems can be compared to puppets that are fully controlled by their users. In contrast, the Body Chat system by Vilhjalmsson and Cassell [48] analyzes the users' text messages in order to automatically generate suitable gestures, facial expressions and eye-gaze behaviors of the avatars participating in a chat. An alternative to video-conferencing is proposed by the Avatar Conference project (www.exodus.gr/Avatar_Conference). In the corresponding system participants are graphically represented by animated 3D depictions of themselves and communicate via a voice channel or via text chat.

Another multi-party application has been proposed by Isbister and colleagues (Isbister et al. [17]). Unlike the above-mentioned applications, their work concentrates on social interaction between several humans in a video chat environment which is supported by a so-called "Helper Agent". Helper Agent is an animated, dog-faced avatar that tracks audio from two-person conversations and intervenes if it detects longer silences.

A system that shares similarities with the Magic Monitor has been proposed by Yabe and colleagues (Yabe et al. [50]). Similar to the Magic Monitor, the content of the dialogue script for the single characters is based on a real conversation between humans. However, they animate discussions from news groups by casting $3 \mathrm{D}$ characters as the authors of the single discussion contributions.

\section{Interactive Performances}

In the scenarios presented so far, the user's role was restricted to select characters for the performance and to set character profiles, but there was no user participation in the performance itself. Rather than playing episodes to be watched by a user, one can try to involve the user actively in a role play. Such 
a scenario, that bears a lot of similarities to improvisational theatre, has been realised in the MIAU project with the development of an interactive version of the eShowroom that has been sketched in Section 5.1.

\subsection{The MIAU Platform}

In this version the user can assign roles to characters, evoke them and watch their car-sales conversations. However, if a user wishes to engage in the carsales conversation, too, s/he can start an avatar that represents her/him in the scenario. As shown in the left-hand screenshot of Fig. 9, the user can participate in the ongoing conversation by typing questions and comments into the text input widget. On the other hand, it is up to the user to decide on the level of active participation. One extreme is that from now on the user asks all questions. In contrast, if the user wants to relax again and just watch the characters talk, s/he can do so, lean back and remain silent. In this case, the virtual buyer will continue to ask questions.

As shown by the architecture in the left-hand part of Fig. 9, the MIAU platform consists of the following components: For each character $\mathrm{C}_{1} \ldots \mathrm{C}_{n}$ MIAU foresees so-called character components containing a separate behavior planner as well as a separate interaction manager. The behavior planner has the task to decompose complex discourse goals into basic acts that can be executed by the character player. Since the user decides on during the performance whether, when and how to participate in a conversation, we cannot start from a pre-defined script. Instead the dialogue between the conversation partners evolves while time progresses. Furthermore, the scenario is openended in the sense that neither the characters nor the users are able to tell what exactly may happen next. The interaction manager, in a certain sense, corresponds to a dialogue manager as found in NL dialogue systems since it is responsible for keeping book on interaction states and the interaction history. However, in the MIAU platform the interaction manager realizes a character's internal interface for communication with other system components by means of formalized communication acts. To this end, the MIAU platform comprises a message board which is shared among the different components for the exchange of internal communication acts.

To allow a user to alter settings for the performance, to take an active part in a performance, or even to intervene in the role of a director or codirector, the platform also incorporates a so-called user component ( $\mathrm{U}$ box in Fig. 9). However, since this time the user decides on what to do, we don't foresee a planner, but an input analyzer for mapping user input onto formalized communication acts. The internal communication with other system components is handled by the interaction manager similar to the structure of a character component. In case the user is represented in the scenario by an embodied (and possibly animated) avatar, the avatar may indicate his or her input activity by means of gestures and speech turns. For instance, if a text widget is used for acquiring user input, the user's avatar will repeat the input 
sentence loudly. Currently, we restrict ourselves to a single participating user only. Nevertheless it seems possible to extend the architecture for multi-user, multi-character scenarios by adding more user components.

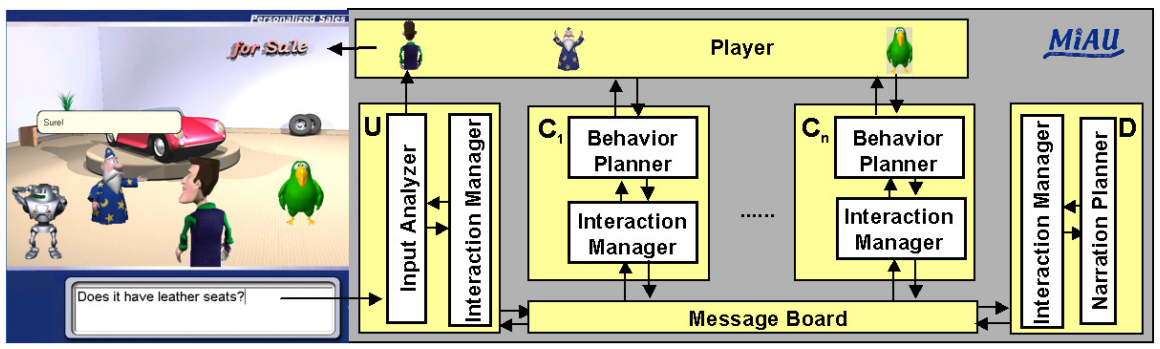

Fig. 9. Screenshot of the interactive version of the eShowroom (left) realized with the MIAU multi-character platform (right)

In addition to character components and the user component, MIAU incorporates also a D-box, the so-called director component. In contrast to the characters, the director does not participate in performances and therefore has no embodiment. Rather, this component is foreseen to enable some degree of centralized control on the overall interactive performance. While the director also comprises a planner, this time the planner is used in order to influence the course of the performance depending on the degree of centralized control wanted for a certain application. Internal communication with other components is again handled via the interaction manager. Examples of flexible switches between different conversational settings (monologue vs. dialog vs. performance vs. interactive performance) can be found in Rist et al. [40].

\subsection{Other Work Related to Interactive Performances}

Even though the focus of interactive drama is usually not on the communication of information, dramaturgical elements offer great promise in presentation scenarios as well, see also Laurel [24]. Our work towards an interactive version of the eShowroom has also been inspired by research on interactive drama that aims at integrating a user in a scenario - either as an audience member or an active participant, see for instance Hayes-Roth et al. [14], Machado et al. [26], and Klesen et al. [22]. To allow for user interaction, some systems incorporate decision points in a narrative-style script (Mateas [29]). That is, the user just decides how to navigate through a graph structure in which nodes represent scenes and edges represent eligible transitions between scenes. In this model any eligible path through the story graph represents a particular plot. In contrast, other approaches rely on story graphs that also comprise special nodes, so-called "freeplay" areas (Sgouros [44]). Within these areas a player 
interacts with one or more characters in complex ways to influence how the story unfolds.

In these approaches characters are usually modeled as autonomous agents that select and instantiate actions under consideration of dramatic constraints, such as the plot of a story or the characters' role and personality (Perlin and Goldberg [35], Hayes-Roth et al. [14]). A key concept of these approaches is improvisation. That is, characters spontaneously and cooperatively work out the details of a story at performance time, taking into account the constraints of directions either coming from the system or a human user.

Depending on the kinds of interactions that occurred in a freeplay area, transitions to follow-up nodes can be enabled or inhibited. Such a mechanism is either realized by means of local evaluation functions for transition selection, or by a so-called plot or drama manager that has global control on the story development (Weyhrauch [49], Mateas and Stern [30]). For instance, the Oz drama manager (Mateas and Stern [30]) selects actions based on an evaluation of the discourse history in terms of dramatic quality. In Sgouros [44] transitions are triggered by events or user/agent actions. In a system for interactive pedagogical drama (Marsella et al. [28]) an evaluation function models pedagogical progress. In the MRE system by Swartout et al. [45], the story graph is called StoryNet. An evaluation function checks a list of conditions, usually tasks that must be completed. To obtain a smooth transition between nodes (scenes), a transition may be associated with an additional pre-scripted scene that is played when the transition is made.

Similarities also exist between the Miau approach and the work described in Cavazza et al. [11] and Young [51]. For the purpose of a computer game application, Cavazza and colleagues use distributed hierarchical planners to control the behavior of multiple characters. In addition, Young introduces an "Execution Manager" that monitors the user's actions and makes realtime decisions about the appropriate system response in case these actions deviate from the planned narrative structure. The Execution Manager roughly corresponds to the Director Component of the MIAU platform.

Another interesting strand of research concerns multimodal conversations with multiple characters. In the context of a military mission rehearsal application Swartout et al. [45] and Traum and Rickel [47] have addressed dialogue management comprising human-character and character-character dialogues in immersive virtual environments. There is no superior component that handles the turn management. To explicitly select the next speaker, the authors have introduced a specific turn-taking action called "Assign-Turn". In this system the characters are realized as completely autonomous agents.

\section{The Interactive CrossTalk Installation}

Seeking for an appealing exhibit that could be demonstrated at the CeBIT 2002 computer fair, the idea arose to have the character Cyberella (cf. Sec- 
tion 4.1) present another existing system, in this case the eShowrrom (cf. Section 5.1). The combination of the two systems resulted in the interactive CrossTalk installation that has been designed as a new variant of information presentation in public spaces, such as an exhibition, or a trade fair.

\subsection{Set-up and Functional View of CrossTalk}

The CrossTalk installation provides a spatially extended interaction experience by offering two separated agent screens, and by creating the illusion that the agents have cross-screen conversations. Figure 10 shows the installation from a distance with two visitors, a close-up of the three screens, and a sketch of the spatial arrangement from a bird's eye view.

In CrossTalk, embodied agents are presented as virtual actors giving interactive performances. This can be considered a playful illustration of the "computers as theatre" paradigm introduced by Laurel [24]. Moreover, the installation relies on what we call a meta-theater metaphor. Quite similar to professional actors, characters in CrossTalk are not always on duty. Rather, they can step out of their roles, and amuse the user with unexpected intermezzi and rehearsal periods. In CrossTalk, embodied agents are presented as virtual actors giving interactive performances.

In CrossTalk Cyberella's primary task is that of a fair hostess. She welcomes visitors who approach the stand and offers them a demonstration of the eShowroom on the opposing screen in which the two virtual actors Tina and Ritchie interchangeably take on the role of a car seller or a potential car buyer and perform car-sales dialogues. The purpose of such simulated sales dialogues is to work through the features of a certain car by means of a question-answering dialogue. Clearly notable variations in the course of such dialogues are due to the specific settings of parameters which can be defined by the user prior to the performance. That is, Cyberella invites the user to assign roles (seller vs. buyer), personality and mood (e.g. polite vs. impolite, agreeable vs. disagreeable), and areas of interest that are associated with cars (safety, comfort, prestige, sportiness, costs, etc.).

Once Cyberella has collected parameter settings from the visitor she takes on the role of a stage director for the actors Tina and Ritchie and instructs them to start the performance. Tina and Ritchie will then change their body postures to signal that they are now "on-duty" and to catch the visitor's attention. Depending on their personality, the agents use different degrees of criticism (customer) and enthusiasm (salesperson) when talking about the car's features (consumption, horsepower, airbags, etc.). During a performance the user can give feedback by pushing one of three buttons ("applause", "boo" and "help"). Such feedback may cause unexpected (meta-theatrical) behavior. For instance, if a visitor submits several times a "boo", the actors will get nervous and may forget their lines. In contrast, "applause" makes them proudly smiling and bowing to the user. When "help" is requested, Cyberella stops the performance for short explanations. 

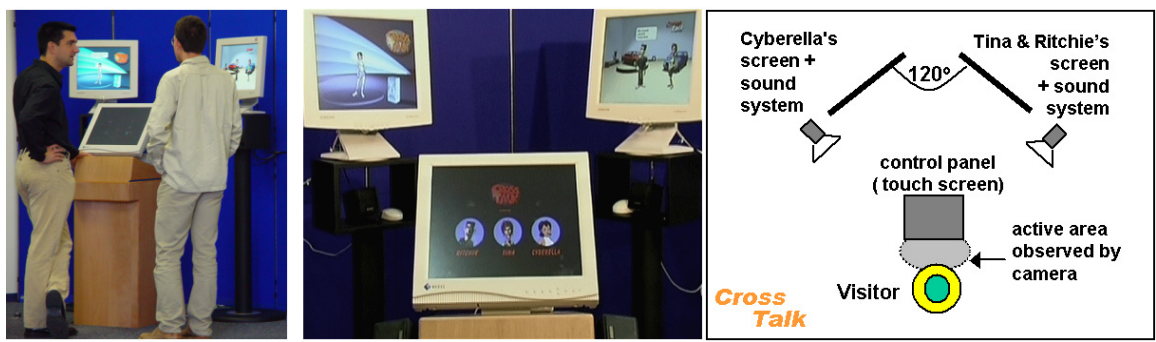

Fig. 10. The interactive installation CrossTalk (cf. http://www.dfki.de/crosstalk)

After the performance, Cyberella takes over again and asks whether the user wants to see another sales dialogue, possibly with new settings. However, if the visitor leaves the installation the actors switch to "off-duty" mode adopting a more relaxed body posture. But instead of just idling around on their screens, the agents display their off-duty behavior by chatting with each other across screens or by "rehearsing" for the next performance. The purpose for this "off-duty" activity, quite natural for humans, is twofold: Firstly, it is a means to encourage leaving visitors to stay for another while watching the "private lives" of the characters and, more important, new potential visitors may be allured from the crowds of passers-by. Secondly, allowing our characters to step out of their roles gives them another human-like quality, one that contributes to the impression that they are permanently alive.

\subsection{Interweaving Scene Authoring with Automated Dialog Generation}

A particular challenge in CrossTalk concerns the scripting of scenes for both the "on-duty" and the "off-duty" mode. In the case of the on-duty mode CrossTalk's subsystem eShowroom is active. As described in Sect. 5.1, the purpose of this system is to inform a user about the features of a car by means of a simulated sales dialogue between a virtual seller and buyer. Such sales conversations can be generated automatically. The car features mentioned in the dialogs, such as a car's consumption, type of engine, etc., are taken from a product database Therefore, the dialogues are just another device to read out stored product information.

To keep the actors alive in the off-duty mode we tried to emulate small talk conversations among stand staff members in case no visitor seems particularly interested in getting information about exhibits. While a broad variation of car sales dialogues can be automatically generated by means of a relatively small number of dialogue patterns, an approach for the automated generation of small talk dialogues (which would be interesting enough for a visitor to listen to) appears much more challenging. We therefore decided to rely on a repertoire of pre-scripted small-talk scenes from which the system would chose randomly when in off-duty mode. For demonstrating CrossTalk at the CeBit 
2002 fair a large corpus of pre-scripted scenes (more than 220 for English and German each) has been assembled by one of the authors with experience in theater acting and directing. Some scenes cover themes related to every-day belongings, such as what to do in the evening, how to get home, or where to get cigarettes. Other scenes refer to the world of the theater or movies. For instance, the characters may reflect on their stagecraft, or what they might do after the CeBIT convention.

The left-hand part of Fig. 11 shows an excerpt of a pre-scripted episode including tags to specify the agent's non-verbal behavior. The specification of such pre-authored dialogues also includes special tags that allow an author to explicitly specify an agent's non-verbal behavior which fall into four categories: gestures, facial expressions, posture shifts and actions. Gestures were taken from a catalogue derived from analyzing a German TV show with manual gesture annotation (Kipp $[19,20])$. The two analyzed speakers were found to have a shared lexicon of 69 conversational gestures of which the most frequently used ones were modelled for CrossTalk by a professional graphics and animation designer and turned into libraries of animation clips. Some facial expressions were added based on the needs of the script (e.g. smile, sceptical). Posture shifts are used to visually separate role (salesperson/customer) and meta-role (actor) of Tina and Ritchie: when they are "themselves as actors" they have a relaxed body posture whereas when they are playacting they straighten up and look more tense. This is in accord with observations in psychotherapy where body posture was found to indicate separate topics or points of view in a conversation (Scheflen [42]). Other actions comprise turning the head, breathing, and other idle-time actions like putting on glasses, yawning etc.
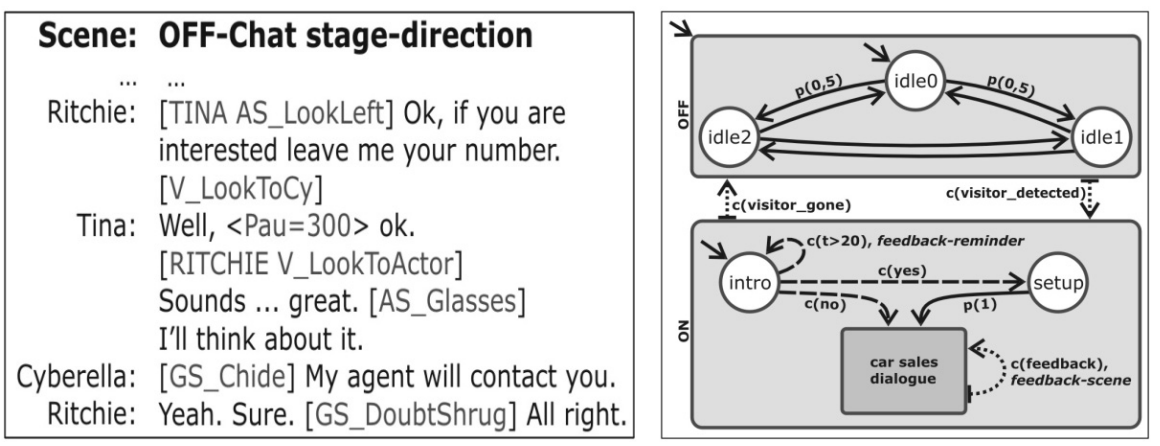

Fig. 11. Excerpt of a pre-scripted scene (left), and excerpt of CrossTalk's scene flow that is defined by a cascaded finite state machine (right)

A simple authoring syntax is meant to appeal to non-programmers, including professional playwrights. The author writes the actors' utterances and includes stage directions in the form of bracketed commands. The script 
can be written in any text processing software. However, to be able to interweave authored and automatically generated scenes at runtime, a script compiler transforms authored scenes into the same internal format that is used by system's presentation planner to represent operators for automated dialog construction.

We realized that apart from the time-consuming scripting effort it is much more of a challenge to create, maintain and extend the overall structure of the evolving story comprising both on-duty and off-duty conversations as well as appropriate responses to potential user feedback. We conceptualize a story as a logical flow of scenes. In CrossTalk scene flow (i.e. the transition between scenes) is modelled by means of a cascaded finite state machine in which states refer to scenes that have been either pre-authored or which are generated automatically on-the-fly. Cascaded finite state machines enable the shared use of modules (part of a scene flow), similar to subroutines in a programming language. This simplifies the modelling process in case of, for instance, repeated patterns of agent-user interactions, such as simple yes/no questions. Scene flows can be specified either in XML syntax or by means of a graphical editor. Scene flow specifications are then compiled into executable finite state machines by the so-called SceneMaker component.

The right-hand part of Fig. 11 shows a small excerpt from CrossTalk's scene flow. After initialization the system starts in off-duty mode. This mode is modelled as a supernode with no scene attached (upper box). The subnode idle0, declared starting node, is processed first. Having performed the attached scene, node $i d l e 1$ or node idle2 are processed with probability 0.5 each. If a visitor arrives, the currently processed node is interrupted by traversing the interrupt edge visitor_detected to supernode on-duty, playing the attached scene intro. Then, Cyberella asks whether the user wants to provide parameters for the CarSales exhibit. This yes/no question is handled with two conditional edges: $c$ (yes) and $c(n o)$. If the user does not answer within a certain amount of time (20 seconds), a third conditional edge $c(t>20)$ is triggered, playing the particular scene named feedback-reminder. During the CarSales performance the user can give feedback by pushing buttons. Multiple interrupt edges $c$ (feedback) handle these button events, interrupting the performance temporarily by playing the respective scenes. Should the visitor leave, the interrupt edge visitor_gone immediately stops all ongoing activities in on-duty mode and enters the off-duty supernode.

\subsection{Towards Self-evolving Characters}

Since the CeBit'02 fair CrossTalk has been exposed at many occasions to several hundreds of people, most of which also interacted with the system. To make the conversations among the characters more interesting and believable we anticipate that the characters should be more adaptive (a) during a session with a particular user, and (b) with regards to previous encounters with other users. A first step in this direction is to collect information 
in a discourse history and to extract from the data key measures like the user's interaction frequency, the user's average response time to questions, types of interaction, quality of feedback (positive, negative, ask for help) as well as the variance in feedback within a session and across users. Informal $\log$ file analysis of CrossTalk sessions showed that it is actually possible to distinguish between several stereotypical user types based on observed interaction patterns. Possible user categories include: critical user (many negative feedbacks), active/passive/slow user (many/few interactions, frequent interaction time-outs), collaborative/obstinate user. Furthermore, feedback can be co-related with particular characters and their actions. For instance, if a user provides more often negative feedback on contributions made by the character Tina but applauds on Ritchie, it can be guessed that this user likes Ritchie more than Tina. In turn, stereotypes as well as unusual interaction patterns can inform the selection of pre-scripted scenes as well as the automated generation of scenes.

With regards to an improvement of the characters' repertoire of potential themes to talk about it is interesting to note that the discourse history represents a rich source of information that can feed small talk conversations among the agents. Rather than relying on pre-scripted small talks (as done in the current CrossTalk system), one may equip the characters with conversation strategies that enable them to reflect on previous encounters. More over, one may give the characters the ability to identify and cluster similar interaction experiences, and to make comparisons and generalizations which in turn can be verbalized in off-duty conversations as well as in a conversation with a user.

CrossTalk also provides an excellent framework to explore the learning of conversational skills. A straightforward approach is to provide the characters with a large number of conversational strategies and correlate the appropriateness of strategies with both, situation of usage and received user feedback. In this way, the characters can automatically acquire and refine criteria and rules for strategy selection. A more ambitious objective is the formation of new strategies. This issue can be approached in several ways. For instance, following an evolutionary approach one can modify (mutate) existing strategies and determine their fitness value in subsequent conversations based on user feedback. In the long run, the characters may also try to acquire new conversational skills from observing and analyzing conversational behaviors of their human interaction partners.

The perhaps most ambitious extension to CrossTalk concerns a somehow automated evolvement of distinct personalities that slowly emerge from one exhibition to another while taking into account interaction experiences with humans and perhaps other virtual characters. However, work in this direction requires more basic research on the simulation of personality development for virtual characters that are embedded in a social context. 


\subsection{Other Work on Installations with Conversational Characters}

A number of installations with interactive characters has been developed by the Synthetic Character Group at the MIT Media Lab. An early piece was the Alive system (Maes et al. [27]) that tracked movements and gestures of a human user to command a virtual dog. Descriptions of more recent installations can be found in Blumberg et al. [8]. While the focus of their work is on expressive character animation to create the illusion of life, CrossTalk characters are enlivened mainly through their engagement in conversations.

Concerning CrossTalk's mixed approach on framing character-character as well as character-human interactions, the work is closely related to the area of interactive drama. Similar to approaches as described in Sect. 7.2 CrossTalk also relies on an authored story graph that frames the flow of scenes. On the other hand, in case a user wants to provide settings for the character's role and mood, the course of car sales performances will depend on this user input.

Finally, the development of new CrossTalk applications comprises basically two authoring tasks. One concerns the specification of the scene flow while the second one concerns the pre-scripting of off-duty scenes and possible intermezzi that may be played in on-duty mode. CrossTalk offers a screenplay-like authoring language to specify the virtual characters verbal and nonverbal behavior as opposed to other approaches that rely on XML-based mark-up languages - some of them can be found in Part 1 of the current book. Though it might be possible to define a scene flow within such mark-up languages, e.g. by using hyperlinks to jump to other parts in the script, CrossTalks clear separation of scene flow definition and in-scene dialogue scripting suggests a systematic breakdown of complex authoring tasks.

\section{Conclusions}

This contribution revisited a number of past and ongoing character systems developed at DFKI. We tried to show that both complexity of conversational setting as well as complexity of underlying architectures are dimensions along which character systems has been evolving. Many of the presented systems make a concrete commitment to a certain conversational setting (monolog vs. dialog vs. presentation teams) and reflect this commitment by a particular system architecture. In contrast to that, the development of the MIAU platform shows that it is indeed possible to develop a single platform which (a) can be used to construct a broad range of character applications, (b) even allows to switch on-the-fly between director- vs. character-centered scripting approaches, and (c) supports a clear separation between the specification of scripting knowledge (being a knowledge-engineering task), and the required computational machinery for behavior generation (being an implementation task). Abstracting from details Miau can be conceived as a reference architecture that has been tailored to applications with conversational characters, and that may be used to systematically compare existing and upcoming systems. 
The presented CrossTalk installation showed that, in order to provide the user with an engaging experience, a compromise has to be made between manual character scripting and automated character control. In CrossTalk such a compromise has been found by interweaving both approaches in two different ways. On the one hand a presentation planner can include authored sub-dialogues in otherwise automatically generated conversations. Vice versa, an authored script may invoke presentation planning to work out a sub-dialog that takes into account certain generation parameters.

As to future work much interesting challenges remain. Robust interpretation of multimodal input is one of them. Further ones concern the improvement of conversational and social skills of characters as well as a character's capability to acquire new skills, e.g. through the deployment of learning mechanisms. Finally, it deserves mentioning that a great deal of research on multimodal communication with virtual characters may be reused in the upcoming generation of conversational robots.

\section{References}

1. André, E., Rist, T.: Coping with temporal constraints in multimedia presentation planning. In: Proc of the AAAI '96, Menlo Park, Cambridge, London (AAAI Press/The MIT Press 1996) pp 142-147

2. André, E., Herzog, G., Rist, T.: Generating multimedia presentations for RoboCup soccer games. In: RoboCup '97: Robot Soccer World Cup I, ed by Kitano (Springer, New York 1997) pp 200-215

3. André, E., Rist, T., Müller, J.: Employing AI methods to control the behavior of animated interface agents. Applied Artificial Intelligence 13:415-448 (1999)

4. André, E., Rist, T., van Mulken, S., Klesen, M., Baldes, S.: The automated design of believable dialogues for animated presentation teams. In: Embodied Conversational Agents, ed by Cassell, J., Sullivan, J., Prevost, S., Churchill, E. (The MIT Press, Cambridge, MA 2000) pp 220-255

5. André, E., Binsted, K., Tanaka-Ishii, K., Luke, S., Herzog, G., Rist, T.: Three RoboCup Simulation League Commentator Systems. AI Magazine 21(1):57-65 (2000)

6. André, E., Baldes, S., Rist, T.: From simulated dialogues to interactive performances. In: Multi-Agent Systems and Applications II, ed by Marik, V., Stepankova, O., Krauwurmovpa, H., Luck, M. (Springer, New York 2002) pp 107-118

7. Binsted, K., Luke, S.: Character design for soccer commentary. In: RoboCup-98: Robot Soccer World Cup II, ed by Asada, M., Kitano, H. (Springer, New York 1999) pp 22-33

8. Blumberg, B., Tomlinson, B., Downie, M.: Multiple conceptions of characterbased interactive installations. Computer Graphics International 2001 (2001) pp 5-11

9. Cassell, J., Pelachaud, C., Badler, N., Steedman, M., Achorn, B., Becket, T., Douville, B., Prevost, S., Stone, M.: Animated conversation: Rule-based generation of facial expression, gesture \& spoken intonation for multiple conversational agents. Computer Graphics 28:413-420 (1994) 
10. Cassell, J., Bickmore, T., Camphell, L., Vilhjalmsson, H., Yan, H.: The human conversation as a system framework: Designing embodied conversational agents. In: Embodied Conversational Agents, ed by Cassell, J., Sullivan, J., Prevost, S., Churchill, E. (The MIT Press, Cambridge MA 2000) pp 29-63

11. Cavazza, M., Charles, F.: Interacting with virtual characters in interactive storytelling. In: Proceedings of AAMAS'02 (2002) pp 318-325

12. Cohen, M.M., Beskow, J., Massaro, DW.: Recent developments in facial animation: An inside view. In: Proceedings of Auditory-Visual Speech Processing 98, Sydney, Australia (1998)

13. Gebhard, P.: Enhancing embodied intelligent agents with affective user modelling. In: Proceedings of UM2001, Doctoral Consortium summary (Springer, Berlin 2001)

14. Hayes-Roth, B., van Gent, R., Huber, D.: Acting in character. Creating personalities for synthetic actors, ed by Trappl, R., Petta, P. (Springer, New York 1997) pp 92-112

15. Höök, K., Persson, P., Sjölinder, M.: Evaluating users' experience of a characterenhanced Information Space. AI Communications 13(3):195-212 (2000)

16. Huber, M.: JAM: A BDI-theoretic mobile agent architecture. In: Proceedings of the 3rd Conference on Autonomous Agents, (ACM Press, New York 1999) pp 236-243

17. Isbister, K., Nakanishi, H., Ishida, T., Nass, C.: Helper agent: Designing an assistant for human-human interaction in a virtual meeting space. In: Proceedings of CHI 2000 (ACM Press, New York 2000) pp 57-64

18. Ishizuka, M., Tsutsui, T., Saeyor, S., Dohi, H., Zong, Y., Prendinger, H.: MPML: A multimodal presentation markup language with character control functions. In: Proceedings of the Workshop on Achieving Human-like Behavior in Interactive Animated Agents, held in conj. with AAMAS'02 (2002) pp 50-54

19. Kipp, M.: From human gesture to synthetic action. In: Proceedings of the Workshop on Multimodal Communication and Context in Embodied Agents, held in conj. with the Fifth International Conference on Autonomous Agents (2001) pp 9-14

20. Kipp, M.: Anvil - a generic annotation tool for multimodal dialogue. In: Proceedings of Eurospeech'01 (2001) pp 1367-1370

21. Kitano, H., Asada, M., Kuniyoshi, Y., Noda, I., Osawa, E., Matsubara, H.: RoboCup: A challenging problem for AI. AI Magazine 18(1):73-85 (1997)

22. Klesen, M., Szatkowski, J., Lehmann, N.: A Dramatised Actant Model for Interactive Improvisational Plays. In: Proceedings of the 3rd Intelligent Virtual Agents Workshop (IVA) (Springer, New York 2001) pp 181-194

23. Kurlander, D., Skelly, T., Salesin, D.: Comic Chat. In: Proceedings of SIGGRAPH'96 (1996) pp 225-236

24. Laurel, B.: Computers as Theatre (Addison-Wesley, Reading MA 1993)

25. Lester. J, Voerman, J.L., Towns, S.G., Callaway, C.B.: Deictic believability: Coordinated gesture, locomotion, and speech in lifelike pedagogical agents. Applied Artificial Intelligence 13:383-414 (1999)

26. Machado, I., Paiva, A., Prada, R.: Is the Wolf Angry or ... Just Hungry? In: Proceedings of Autonomous Agents (ACM Press, New York 2001), pp 370-376

27. Maes, P., Darrell, T., Blumberg, B., Pentland, A.: The ALIVE system: Wireless, full-Body Interaction with autonomous agents. In: ACM Special Issue on Multimedia and Multisensory Virtual Worlds (1996) 
28. Marsella, S.C., Johnson, W.L., LaBore, C.: Interactive pedagogical drama. In: Proceedings of the Fourth International Conference on Autonomous Agents (ACM Press 2000) pp 301-308

29. Mateas, M.: An Oz-Centric review of interactive drama and believable agents. Technical Report CMU-CS-97-156, School of CS, CMU, Pittsburgh, PA. (1997)

30. Mateas, M., Stern, A.: Towards integrating plot and character for interactive drama. In: Working notes of the Social Intelligent Agents: The Human in the Loop Symposium (AAAI Press 2000)

31. Matsubara, H., Frank, I., Tanaka-Ishii, K., Noda, I., Nakashima, H., Hasida, K.: Character design for soccer commentary. In: RoboCup-98: Robot Soccer World Cup II, ed by Asada, M., Kitano, H. (Springer, New York 1999) pp 34-49

32. Nitta, K., Hasegawa, O., Akiba, T., Kamishima, T., Kurita, T., Hayamizu, S., Itoh, K., Ishizuka, M., Dohi, H., Okamura, M.: An experimental multimodal disputation system. In: Proceedings of the IJCAI'97 Workshop on Intelligent Multimodal Systems (1997)

33. Noma, T., Badler, N.: A virtual human presenter. In: Proceedings of the IJCAI '97 Workshop on Animated Interface Agents: Making them ntelligent (1997) pp $45-51$

34. Pelachaud, C., Carofiglio, V., De Carolis, B., de Rosis, F., Poggi, I.: Embodied contextual agent in information delivering application. Proceedings of $A A$ MAS'02 (ACM Press, New York 2002) pp 758-765

35. Perlin, K., Goldberg, A.: Improv: a system for scripting interactive actors in virtual worlds. Computer Graphics 30:205-216 (1996)

36. Prendinger, H., Ishizuka, M.: Social role awareness in animated agents. In: Proceedings 5th International Conference on Autonomous Agents (Agents-01) (ACM Press, New York 2001) pp 270-277

37. Rich, C., Sidner, C.L., Lesh, N.: COLLAGEN: Applying collaborative discourse theory to human-computer interaction. AI Magazine 22(4):15-25 (2001)

38. Rickel, J., Johnson, W.L.: Virtual humans for team training in virtual reality. In: Proceedings of the Ninth International Conference on Artificial Intelligence in Education (IOS Press, Amsterdam 1999) pp 578-585

39. Rist, T., André, E., Müller, J.: Adding animated presentation agents to the interface. Proceedings of IUI 97 (ACM Press, New York 1997) pp 79-86

40. Rist, T., André, E., Baldes, S.: A flexible platform for building applications with life-Like characters. In: Proceedings of IUI'03 (ACM Press 2003) pp 158-165

41. Rist, T., Schmitt, M.: Applying socio-psychological concepts of cognitive consistency to negotiation dialog scenarios with embodied conversational characters. Proceedings of AISB'02 Symposium on Animated Expressive Characters for Social Interactions (2002) pp 79-84 (Extended version submitted for publication in 2003)

42. Scheflen, A.E.: The significance of posture in communication systems. Psychiatry 26:316-331 (1964)

43. Smith, M.A., Farnham, S., Drucker, S.M.: The social life of small graphical chat spaces In: Proceedings of CHI'2000 (2000) pp 462-469

44. Sgouros, N.: Dynamic generation, management and resolution of interactive plots. Artificial Intelligence 107(1):29-62 (1999)

45. Swartout, W., Hill, R., Gratch, J., Johnson, W.L., Kyriakakis, C., LaBore, C., Lindheim, R., Marsella, S., Miraglia, D., Moore, B., Morie, J., Rickel, J., Thiébaux, M., Tuch, L., Whitney, R., Douglas, J.: Toward the Holodeck: Integrating graphics, sound, character and story. In: Proceedings 5th International 
Conference on Autonomous Agents (Agents-01) (ACM Press, New York 2001) pp 409-416

46. Thalmann, N.M., Kalra, P.: The simulation of a virtual TV presenter. In: Computer graphics and applications (World Scientific Press, Singapore 1995) pp 9-21

47. Traum, D., Rickel, J.: Embodied agents for multi-party dialogue in immersive virtual worlds. In: Proc of AAMAS'02 (ACM Press, New York 2002) pp 766-733

48. Vilhjalmsson, H., Cassell, J.: BodyChat: Autonomous Communicative Behaviors in Avatars. In: Proceedings of Autonomous Agents'98 (1998) pp 269-276

49. Weyhrauch, P.: Guiding interactive drama. Ph.D. thesis. Carnegie Mellon University (1997)

50. Yabe, J., Takahashi, S., Shibayama, E.: Automatic animation of discussions in usenet. In: Proceedings of Advanced Visual Interfaces 2000 (2002) pp 84-91

51. Young, M.: The cooperative contract in interactive entertainment. In: Socially Intelligent Agents: Creating Relationships with Computers and Robots, ed by Dautenhahn, K., Bond, A.H., Canamero, L., Edmonds, B. (Kluwer, Dordrecht 2002) pp 229-234

52. Wahlster, W., Reithinger, N., Blocher, A.: SmartKom: Multimodal communication with a life-Like character. In: Proceedings of EUROSPEECH'01 (2001)

53. Walker, M.A., Cahn, J.E., Whittacker, S.J.: Improvising linguistic style: Social and affective bases for agent personality. In: Proceedings First International Conference on Autonomous Agents (Agents'97) (ACM Press, New York 1997) pp 10-17 